Théologiques

Revue interdisciplinaire d'études religieuses

\title{
Pour une culture palliative ?
}

Fragilités et vulnérabillité

\section{Jean-Marc Barreau}

Volume 28, numéro 1, 2020

Pour une culture palliative ? Fragilités et vulnérabilité

URI : https://id.erudit.org/iderudit/1074673ar

DOI : https://doi.org/10.7202/1074673ar

Aller au sommaire du numéro

Éditeur(s)

Institut d'études religieuses de l'Université de Montréal

ISSN

1188-7109 (imprimé)

1492-1413 (numérique)

Découvrir la revue

Citer ce document

Barreau, J-M. (2020). Pour une culture palliative ? Fragilités et vulnérabilité.

Théologiques, 28(1), 7-16. https://doi.org/10.7202/1074673ar d'utilisation que vous pouvez consulter en ligne.

https://apropos.erudit.org/fr/usagers/politique-dutilisation/ 


\title{
Pour une culture palliative?
}

\section{Fragilités et vulnérabilité}

\author{
Jean-Marc BARREAU* \\ Institut d'études religieuses \\ Université de Montréal (Canada)
}

"Le Mouvement moderne des Hospices» (Lamau 2014) a pour genèse l'ouverture du St Christopher's Hospice, à Sydenham, au sud-est de Londres. Un mouvement qui se démarque par sa rapide croissance ainsi que par son enracinement dans une longue tradition des soins hospitaliers. En effet, en janvier 1975, « le Dr Balfour Mount ouvrait une Unité de Soins palliatifs au Royal Victoria Hospital de Montréal, Québec » quand un premier groupe de professionnels de la santé «fondait à New Haven l'Hospice Incorporatel of Connecticut » (Lamau 2014) ${ }^{1}$. Et si en juin 1980, 17 pays participent à l'International Hospice Conference à Londres, au décès de Cicely Saunder, en 2005, les soins palliatifs sont alors pratiqués dans plus de 120 pays (Lamau 2014). Cette rapide expansion doit son succès — deuxième point - à une longue tradition des soins hospitaliers où la réalité qu'ils désignent est antérieure à une dénomination précise (Ricot 2016). C’est le cas par exemple pour la France, où la tradition palliative précède largement la création officielle des soins palliatifs en 1986 par la promulgation d'une circulaire ministérielle (Lamau 2014). Une antériorité qui s'explique no-

* Professeur adjoint, Jean-Marc Barreau est aujourd'hui spécialiste de l'intervention spirituelle. Son cursus, riche à la fois d'un solide ancrage clinique et d'une expertise spécifique en anthropologie spirituelle, l'y a longuement préparé. Ses recherches académiques actuelles se concentrent notamment sur la complexité du processus du deuil et sur les diverses modalités de son accompagnement spirituel.

1 Nous renvoyons ici à l'article de Doucet dans ce numéro de Théologiques : quoique traitant son sujet d'un point de vue éthique, il traverse l'origine et l'historique des soins palliatifs.

(C) Revue Théologiques 2020. Tout droit réservé. 
tamment par la culture hospitalière que les institutions religieuses ont soigneusement entretenue. De fait, « [leur] souci ne se réduisait pas à préparer les moribonds à une mort chrétienne, mais visait aussi à adoucir les vies des personnes vulnérables et pas nécessairement en fin de vie. On songe ici au rôle de pionnier joué au XIX ${ }^{\mathrm{e}}$ siècle [en France] par l'institution Jeanne Garnier » (Ricot 2016, 49). Veuve à l'âge de 24 ans, cette jeune femme fondera l'CEuvre du Calvaire qui aura pour vocation d'accueillir les déboutés des hôpitaux, parce qu'incurables. Difficile de ne pas voir dans cette tradition un point commun avec la Société Notre-Dame de Montréal que Monsieur Jérôme le Royer de la Dauversière fondait deux siècles auparavant, accréditant une Jeanne Mance jusqu'en Nouvelle-France.

Si cet exorde nous rend sensibles à la rapide croissance des soins palliatifs et de «la science palliative» (Barreau 2017), il désigne l'enracinement historique de la culture palliative comme l'une des causes de cette célérité. Distinguée par sa capacité à transcender la dualité entre la sphère séculière et le monde religieux - Jeanne Garnier n'a jamais fondé de congrégation religieuse et Jeanne Mance non plus -, cette culture se démarque surtout par sa capacité à définir une épistémologie de soins très spécifique. Ces soins qui « n'ont pas comme vocation première la guérison d'une pathologie grave risquant d'emporter le malade » (Ricot 2016, 50) se démarquent par un accompagnement holistique de la personne dans toutes ses fragilités.

Ceci étant convenu, nous pouvons maintenant indiquer au lecteur quelles perspectives ce numéro de Théologiques annonce par son titre et par son sous-titre. Au risque d'étonner, bien que l'article de Barreau et Cara offre une analyse rigoureuse de ce qu'est la science palliative, "Pour une culture palliative? » n'a jamais prétendu constituer un traité scientifique ou une synthèse historique précisant ce qu'est la science palliative, ni non plus définir ce qu'est la médecine palliative ${ }^{2}$. La visée de ce numéro de Théologiques est plutôt de mettre à jour la spécificité de la culture palliative, nourricière par capillarité axiologique de l'épistémologie des soins palliatifs. N'est-ce pas là d'ailleurs l'une des constantes incombant à toute démarche scientifique que de nommer et circonscrire chacun des soubassements sémantiques de l'objet analysé ? Ici, nous pensons que cette culture

2 Nous renvoyons là encore le lecteur à l'article de Doucet qui, dans ce numéro de Théologiques, souligne toute l'ambiguité du terme « médecine palliative». 
palliative se distingue dans sa capacité à visiter les fragilités humaines jusqu’à les assumer : "Fragilités et vulnérabilité » tel est bien notre soustitre. C'est donc bien cette vision qui offre son unité sémantique à notre publication - jusqu'à venir substituer in fine le point d'interrogation originellement prévu par un point d'exclamation. D'ailleurs, un rapide état de la question vient confirmer ce regard. Car si de nombreux ouvrages, et certains d'une grande qualité d'analyse ${ }^{3}$, offrent une typologie des fragilités humaines, moins nombreux sont ceux qui abordent la question de la « vulnérabilité ${ }^{4} »$ humaine.

C'est donc bien autour de cette question que nous avons voulu construire ce numéro de Théologiques, en faisant ultimement de ce liminaire un lieu de dialogue entre les différents auteurs ${ }^{5}$ qui ont participé à son projet de publication : Allard; Barreau et Cara; De Koninck; Doucet; Leboucher ; Lemieux ; Saucier. Auteurs que nous nous empressons de remercier très sincèrement, et pour le temps qu'ils ont pris à rédiger leurs textes, et pour la qualité de ces derniers.

\section{Fragilités}

Je considère que la citation subséquente de François Mauriac unifie parfaitement l'article de Leboucher, tel son centre d'inertie : "C'est une étrange injustice que de faire grief au chrétien du sentiment qu'il a de sa propre misère, et de l'accuser d'être faible parce qu'il se connaît comme tel. Il n'y a pas de courage à ne pas se connaître; mais il y en a beaucoup à se regarder en face » (Mauriac 1949, 34-35). La pertinence de ces propos ne tient pas nécessairement à leurs références chrétiennes - bien que nous reviendrons sur ce point plus en avant -, mais davantage au devoir qu'a tout humain de faire face à ce qu'il est réellement. Ces mots n'affirment-ils pas sans ambages que se reconnaître fragile n'est pas signe de faiblesse, bien au contraire? La démarche de Leboucher s'inscrit sans l'ombre d'un doute dans le champ de l'anthropologie culturelle. Sylvie Germain, Nicolas de Staël et

3 Pour ne citer que ceux-ci : «Fragilité » de Jean-Louis Chrétien (2017); «Le souffle et le roseau, Variations sur la fragilité » de Marc Leboucher (2017) ; "Fragile asile » de Véronique Dufief (2019)

4 Citons néanmoins l'excellent ouvrage : «Vulnérabilité. Pour une philosophie du soin » de Jean-Philippe Pierron (2010).

5 L'utilisation exclusive du masculin a pour seul but d'alléger le texte. 
Glenn Gould apparaissent tour à tour comme des témoins privilégiés de « la fragilité dans la culture ». Écrivain, peintre ou pianiste de renom, leurs rapports éminemment personnels et «charnels » à l'œuvre «en devenir de...» font émerger au cœur des sinuosités de la culture contemporaine les nervures essentielles des fragilités d'ici et maintenant. Pour autant, l'auteur des Variations sur la fragilité ne cherche pas à sanctuariser « le fragile». Le sujet est bien plus profond : il s'agit de reconnaître à partir des fragilités les pierres angulaires permettant la mise à jour des «attentes spirituelles » d'aujourd'hui et de demain.

Le nid de la ou des spiritualités se situerait-il donc au creux de la fragilité de chacun? À la différence du « troisième homme » introduit par Leboucher, celui issu de la modernité se situant à l'orée de la postmodernité, l'artiste fait donc l'expérience de ces craquèlements intérieurs révélant la friabilité de la matière. Lui-même matière en transformation, il en devient une icône vivante...

Fidèles à nos liens avec François Mauriac et à sa référence à l'éthique chrétienne, nous souhaitons rejoindre Lemieux : "Ultime fragilité et sublime espérance, l'expérience de la mort. » La mort et son annonce sont par définition l'ultime fragilité provoquant franchement le troisième homme non seulement parce qu'on ne la connait pas - on ne peut donc pas l'anticiper - mais aussi parce qu'on n'en revient pas - on ne peut donc pas la réparer. Mais pour reprendre les mots de Lemieux et sauvegarder l'intuition de François Mauriac, nous comprenons qu'ultimement le chrétien ait l'audace de se regarder face à sa mort, car dans l'espérance chrétienne il y a "un programme sémiotique ». Si avec Péguy, nous comprenons que « la petite fille espérance » s'entoure de la foi et de la charité, il n'en reste pas moins vrai que seule elle aura l'élan nécessaire pour faire reconnaître à l'horizon de la mort le «signe » de l'ultime fragilité humaine déjà transfigurée par l'Autre. " Mourir, espérer, vivre. » Voici donc le triptyque de l'article du théologien qui, pour reprendre les mots mêmes de son auteur, vit dans la misère puisqu'il a la «faiblesse de croire » (expression reprise de De Certeau). Si la réalité de la mort y est présentée à la fois par une analyse historique et anthropologique, nous retenons ici que seule « [l']ultime fragilité que révèle l'expérience de la mort, appelle [...] une sublime espérance ». François Mauriac ne canonise donc pas tous les chrétiens, mais il rappelle le cœur de l'éthique chrétienne, cette grâce divine qui cherche son «enclos»dans la finitude humaine afin de mieux l'en 
« sortir » pour reprendre une expression souvent utilisée par Lemieux. Car l'espérance « s'avère être une véritable éthique de la marche ». Dès lors, que nous soyons des cliniciens ou des théologiens, des intellectuels ou des praticiens, des proches aidants ou des frères en humanité, des agnostiques ou des athées, mais aussi bien évidemment des croyants, nous osons l'audace de cette fragilité « visitée » : la nommer, c’est déjà prendre le chemin...

En ce sens, l'article de Barreau et de Cara nous fait faire un pas décisif en avant dans la prise en compte d'une typologie de la fragilité humaine. Égrainant un à un les différents niveaux d'isolement que le patient peut vivre $^{6}$, « l'isolement sanitaire ", « l'isolement social », « l'isolement culturel », " l'isolement spirituel », " l'isolement religieux » — échos de notre postmodernité - l'analyse scientifique des deux auteurs associe à chaque type d'isolement un type particulier de fragilités. Toutes ont en commun une brisure d'altérité... Comment ne pas dès lors souhaiter avec Lemieux que « l'espérance germe dans les profondeurs de ce gouffre »? Et pour que cela se réalise, ne faut-il pas que le passage s'opère, ouvrant nos «fragilités » au paradigme de la «vulnérabilité »?

\section{Vulnérabilité}

Dans son article, Leboucher pose directement la question : "Comment passer d'une fragilité initiale à une vulnérabilité consentie et partagée?» S’il nous rappelle que chez Levinas « le visage exprime la vulnérabilité de l'autre et invite à la responsabilité à son égard », il tend ainsi la main à la réflexion conduite par Allard. Le texte de celle-ci n'est pas seulement une « Réflexion sur les fragilités vécues par les proches aidant(e)s », il est surtout la preuve du passage possible et souhaité entre le monde des fragilités et celui de la vulnérabilité. Citant Nadeau et son ouvrage À l'école de la fragilité (2019), Allard rappelle un point central sur le plan anthropologique : la fragilité n'est jamais seule, puisqu'elle est accompagnée par des forces intrinsèques à la personne. La question de l'accompagnement sera le prochain point abordé par ce liminaire. Ici, nous souhaitons précisément rete-

6 L'article s'appuie sur une étude de cas en pleine période de pandémie de la Covid-19. Mais la question de l'isolement sanitaire peut se poser pour toute pandémie comme d'ailleurs malheureusement pour « toute mise à l'écart » du patient ou de la personne qui se reconnaît fragile et qui n'est pas reçu dans cette réalité. 
nir cette réalité anthropologique : la personne accompagnée, le patient accompagné, l'agonisant lui aussi accompagné, tous " traversent » leurs fragilités, transfigurés par l'espoir, pour certains par l'espérance. Parmi les attentes spirituelles contemporaines développées par Leboucher, l'une d'elles rejoint explicitement l'analyse d'Allard : il s'agit de la gratuité. Estil possible que ces deux auteurs pointent « l'espacement » anthropologique (Barreau 2015,148 ) au cœur duquel le passage des fragilités à la vulnérabilité peut s'opérer? N'est-ce pas là le lieu d'une conversion épistémologique, interrogera Lemieux? D'ailleurs, si notre sous-titre parle au pluriel des fragilités et au singulier de la vulnérabilité, c'est parce que l'homme n'est pas intrinsèquement fragile quoique porteur de fragilités, alors que l'homme n'a pas des vulnérabilités, il est vulnérable. D’un point de vue ontologique, la fragilité demeure un "accident » alors que la vulnérabilité et son acolyte, la gratuité, s'invitent au rang de l'essentiel.

Cette notion de gratuité en vis-à-vis de la vulnérabilité place le rapport du patient et du proche aidant dans une dimension de corporéité (Barreau 2014, 91), car c'est dans un seul corps axiologique qu'ils cheminent ensemble. C'est alors par « capillarité » (Barreau 2014, 98-99) que la quête de sens peut opérer. Il n'y a pas en haut le proche aidant et en bas le patient. Allard le dit bien : «[...] cette conscience de la fragilité humaine permet cette présence vraie et authentique aux côtés de l'autre en situation de fragilité ». La "vulnérabilité circulaire » (Barreau 2017, 47-52) reprise par les articles de Allard et Leboucher se fait ainsi dynamique de vie. N'y aurait-il pas là l'un des fondements possibles pour une spiritualité anthropologique ? Mais au sujet de la vulnérabilité, l'article de Saucier offre un paradigme tout autre, bien que toujours à partir du rapport de l'homme à sa fragilité, ici la mort. Ce vis-à-vis radical est la complexe et souvent difficile rencontre de l'homme avec lui-même. "Le deuil du Moi en Soi devant la mort annoncée » déplace le rapport temporel usuel à la mort et la manière d'entrer dans une vulnérabilité humaine assumée. La thèse défendue par Saucier est que la mort annoncée « oblige » le patient à une libération du Moi. Les fragilités regarderaient le Moi alors que la vulnérabilité serait d'ordre existentiel. Dès lors, pour l'auteur, « cette vulnérabilité de la personne ne met pas en jeu que son intégrité physique, mais aussi toutes les dimensions de son Être global ». Saucier résumera parfaitement son analyse dans le dénouement de son article : "Ce deuil inachevable s'effectue par un mouvement de retraite du Moi rendu nécessaire par la fragilité du 
corps, pour ensuite répondre à la vulnérabilité de l'Être par la révélation du Soi. »

\section{Accompagnement}

Quand Saucier place en vis-à-vis le Soi et le Moi, dans une émergence du Soi tel un phare battu par la tempête, il interpelle nécessairement le paysage océanique de l'accompagnement. Cet anéantissement du Moi, matérialisé par l'écume échouée au pied du phare, peut-il s'opérer par luimême? Sinon au regard de quel type de posture de la part de l'accompagnateur ? C'est là le défi majeur posé dans le cadre de cet article qui s'inscrit délibérément dans un autre paradigme que celui offert par De Koninck sur l'ethos palliatif. Et Saucier ne s'en cache pas : « La dynamique interne entre le système d'inertie, la souffrance et le travail du deuil, recherche davantage la flexibilité d'accompagnement qu'une résolution normative. » Dans cette science de l'accompagnement, il y a donc bien un double vis-à-vis à considérer : celui du Soi face au Moi et celui de l'accompagnateur en pourparlers thérapeutiques avec le patient vulnérable. Lacan lui-même, dont les référents conceptuels traversent la troisième partie de l'article de Lemieux, ne considère-t-il pas que la "destinée ultime » du thérapeute ou de l'analyste - pour nous l'accompagnateur — est «vouée au final à être rejetée tel un déchet »? (Veilleux 2020, 16).

Dans ce jeu sémantique de postures à Soi ou à l'autre et/ou à l'Autre, l'article de Doucet « De la douleur totale à la souffrance existentielle » interpelle à son tour la « science de l'accompagnement » (Barreau 2017), mais sous un autre angle, éthique. Reprenant l'historique des soins palliatifs tout en faisant mémoire de l'œuvre de Cicely Saunders, l'éthicien pose deux questions fondamentales : « Pourquoi ne pas intégrer la souffrance existentielle à la réalité de la douleur totale? » « Pourquoi ne pas associer à la souffrance existentielle la souffrance spirituelle?» Si personnellement j'ai voulu planter ces deux questions au cour de cette réflexion sur l'accompagnement, c'est pour offrir au lecteur une autre prise de vue possible sur cette question aussi cruciale que délicate, mais aussi pour rappeler que la «science palliative » est bien celle de l'accompagnement. Que veut dire intervenir? Que signifie accompagner? Et quid de l'accompagnement spirituel? Et de la souffrance spirituelle? 
Si la «souffrance existentielle » et la « souffrance spirituelle » restent encore à ce jour en décalage avec un certain type de médecine palliative, il est possible que la réponse à ce hiatus nous soit donnée par les deux compagnons de « route » proposés par Lemieux : le «blessé » et le «bon samaritain ». Nous avons dans cette rencontre tout le paradoxe, mais aussi toute la richesse du lien qui se propose à nous entre l'intervention spirituelle et l'accompagnement spirituel. Quand l'intervention s'engage à guérir le blessé, l'accompagnement consiste à marcher "gratuitement» et dans la "vulnérabilité » auprès de l'autre que soi. Dès lors, ne faut-il pas accepter que l'accompagnement ne puisse pas soulager parfaitement la souffrance? La souffrance existentielle? La souffrance spirituelle? Le concept «d'impuissance spirituelle » introduit par Barreau et Cara renvoie à cette terrible réalité : s'il est du devoir de l'intervenant, du thérapeute, du clinicien et de l'éthicien, de tout faire pour soulager dignement la douleur et apaiser chaque souffrance - c'est là un dogme de l'humanisme -, il n'en est pas moins vrai que la souffrance, ainsi qu'ultimement la mort, laissent trop souvent l'accompagnateur impuissant du fait même qu'elles s'imposent. N'est-ce pas là une réalité qui bouscule l'hégémonie médicale? N'est-ce pas ce qui interpelle la science palliative et son épistémologie ? Ricot nous prévient quand il affirme que les soins palliatifs « naissent de l'aveu de l'impuissance à guérir, du consentement à la finitude humaine, de l'humilité devant le tragique de la condition mortelle »(Ricot 2016, 50). Pourtant, "cette faiblesse assumée est la condition d'une nouvelle puissance, celle d'offrir à la vie, y compris à ses derniers moments, les conditions d'un accomplissement et le réconfort d'un accompagnement»(Ricot 2016, $50)$.

\section{$4 \quad$ L'etbos palliatif}

Il convient donc maintenant de répondre à la question initiale qui porte cette publication : "Pour une culture palliative?» Dans son article, quand De Koninck traite de "l'ethos palliatif », il renvoie directement à Levinas et à Kant - pour souligner le fait que le visage ne se dévoile vraiment que dans les yeux de l'autre, ce qui situe les deux vis-à-vis dans ce que j'ai déjà nommé "la vulnérabilité circulaire »; et pour distinguer dignité et prix. "Ce qui a du prix peut être remplacé », rappelle le philosophe, alors que ce qui est «supérieur à tout prix se nomme la dignité ». La culture palliative dépendrait donc de la capacité de l'être humain fragilisé à s'en 
remettre dignement à son vis-à-vis ? Et vice versa ? N'est-ce pas là la raison pour laquelle De Koninck qualifie les professionnels en soins palliatifs « d'experts en humanité »? Et n'est-ce pas là encore la raison pour laquelle De Koninck définit l'ethos palliatif à travers les mots de Marie de Hennezel quand elle souligne « le défi que pose la mort d'autrui » ? La culture palliative serait donc bien cette capacité à accompagner les innombrables fragilités humaines, ultimement la mort. À partir de la vie et pour la vie... Le toucher thérapeutique dont parle De Koninck, dûment qualifié par une déontologie professionnelle scrupuleuse, n'est plus un simple «traitement ». Il devient un toucher de vie... Celui-là même qui apaise la souffrance, j'espère celle existentielle, sinon celle spirituelle.

Attentive aux visages « parcheminés » et aux mains «fendillées », la culture palliative est donc celle d'un corps vivant, fort de ses fragilités et riche de sa vulnérabilité, dont la vocation est d'offrir la vie par simple capillarité axiologique. Une culture où le bon samaritain respecte sur son chemin les cailloux blancs balisant les pourtours d'un humanisme intégral : l'écoute, la présence, la gratuité, la dignité, l'altérité, les fragilités, la quête de sens, la vulnérabilité circulaire, la compassion...

Une sorte d'écologie intégrale où la protection des plus fragiles, mais aussi « du plus fragile » en chaque personne, engage nos cultures et notre éthique, interpelle notre recherche scientifique interdisciplinaire voire transdisciplinaire sur ce qu'est l'intégralité de la personne et sur ce qu'est son devenir vital... La dimension bigarrée de cette publication renvoie donc bien à la fois à la richesse et à la diversité de cette culture palliative. Dès lors, « Pour une culture palliative! » souligne l'exigence — ou l'urgence - de préciser les anthropologies qui la sous-tendent dans le but de mettre à jour une épistémologie des « soins palliatifs » qui soit respectueuse de chacun de ses cailloux blancs, ultimement de celui qu'est « la vulnérabilité circulaire ».

\section{Références}

Barreau, J.-M. (2017), Soins palliatifs. Accompagner pour vivre !, Paris, Médiaspaul. (2015), François et la miséricorde, Paris, Médiaspaul. (2014), Jean-Paul II. Le saint de la nouvelle évangélisation, Paris, Salvator. 
ChrÉTIEN, J.-L., (2017), Fragilités, Paris, Éditions de Minuit.

Doucet, H. (2014), L'éthique clinique. Pour une approche relationnelle dans les soins, Montréal, PUM.

DUfief, V. (2019), Fragile agile, Paris, Salvator.

Lamau, M.-L. (2014), " Origine et inspiration, Cicely Saunders à la naissance des soins palliatifs ", Revue d'éthique et de théologie morale, $\mathrm{n}^{\circ}$ 282, p. 55-81.

https://www.cairn.info/revue-d-ethique-et-de-theologie-morale2014-5-page-55.htm

LEBOUCHER, M. (2017), Le souffle et le roseau, Variations sur la fragilité, Paris, Salvator.

Ricot, J. (2016), "Histoire et éthique des soins palliatifs », Cités, n ${ }^{\circ}$ 66, p. 49-58. https:/www.cairn.info/revue-cites-2016-2-page-49.htm

VeILleuX, M. (2020), À la lumière du séminaire XVII de Jacques Lacan : Dans quel discours se tient (ou est pris) l'intervenant en soins spirituel? Essai, Maîtrise en théologie pratique, sous la direction de M. Guy-Robert Saint-Arnaud, IÉR., FAS, Université de Montréal. 